BINP 93-88

October 1993

\title{
Magnetic quadrupole moment of $W$-boson in Kobayashi-Maskawa model
}

\author{
I.B. Khriplovich' and M.E. Pospelov' \\ Budker Institute of Nuclear Physics, 630090 Novosibirsk
}

\begin{abstract}
Due to CP-invariance violation a vector particle can acquire $\mathrm{T}$ - and P-odd electromagnetic moment, magnetic quadrupole one. The $\mathrm{W}$-boson magnetic quadrupole moment is calculated in the Kobayashi-Maskawa model. This is the only known $\mathrm{CP}$-odd moment arising in this model in two-loop approximation.
\end{abstract}

\footnotetext{
${ }^{1}$ khriplovich@inp.nsk.su

${ }^{2}$ pospelov@inp.nsk.su
} 
1. The Kobayashi-Maskawa (KM) model looks now as the most natural description of CP-violation. It describes properly CP-odd phenomena in the decays of neutral $K$ mesons and predicts extremely tiny CP-odd effects in the flavour-conserving processes. Though its predictions for the electric dipole moments (EDM) of elementary particles are far beyond the present experimental facilities, the corresponding theoretical investigations are of certain methodological interest.

To lowest, one-loop approximation in the weak interaction all CP-odd flavour-conserving amplitudes in the KM model turn to zero trivially. The point is that in this approximation those amplitudes depend only on the moduli squared of elements of the KM matrix, so the result cannot contain the $\mathrm{CP}$-violating phase.

However, the EDMs of a quark and W-boson vanish also to next, two-loop approximation as well[1, 2]. It has no transparent explanation, though in Ref. [3] an attempt was made to explain vanishing of the W-boson EDM in two-loop approximation starting from general principles only.

In the present work the W-boson magnetic quadrupole moment (MQM) is calculated in the KM model. It arises already in two-loop approximation which means in particular that the vanishing of EDM in this approximation is a very specific, dynamic phenomenon only.

To avoid a possible misunderstanding we wish to mention here that the interaction of the W-boson MQM with an external electromagnetic field, being of second order in the field momentum $q$, does not induce for instance the electron EDM since its interaction with an electromagnetic field is of first order in $q$.

2. A general consideration ascending to Ref. [u] demonstrates that a particle of spin $I$ can have $2 I \mathrm{P}$ - and T-odd electromagnetic moments. In particular, at $I=1$ there are two such moments. Besides an electric dipole moment a particle of spin one can have a magnetic quadrupole moment.

We define the MQM operator by analogy with the electric quadrupole one, via the interaction with the corresponding field gradient:

$$
\begin{aligned}
\hat{H}_{Q} & =-\frac{1}{6} \hat{Q}_{i j} \nabla_{i} E_{j}, \\
\hat{H}_{M} & =-\frac{1}{6} \hat{M}_{i j} \nabla_{i} B_{j} .
\end{aligned}
$$

The tensor $\hat{M}_{i j}$ is expressed as usual via the irreducible second-rank tensor constructed from the spin operator $\hat{I}_{i}$ :

$$
\hat{M}_{i j}=\mathcal{M} \frac{3}{2 I(2 I-1)}\left[\hat{I}_{i} \hat{I}_{j}+\hat{I}_{j} \hat{I}_{i}-\frac{2}{3} \delta_{i j} I(I+1)\right] .
$$

The expectation value $\mathcal{M}$ of the operator $\hat{M}_{z z}$ in the state with the maximum spin projection $I_{z}=I$ will be called magnetic quadrupole moment.

Let us consider now the effective interaction of the W-boson with magnetic field gradient. Since the spin operator acts as follows on the polarization vector e of a vector particle 
at rest [5]:

$$
\left(\hat{I}_{i} \mathbf{e}\right)_{\mathbf{k}}=-\mathbf{i} \epsilon_{\mathbf{i k l}} \mathbf{e}_{\mathbf{l}}
$$

for such a particle the matrix element of the MQM interaction reduces to

$$
H=\frac{1}{4} \mathcal{M} e_{i}^{*} e_{j}\left(\nabla_{i} B_{j}+\nabla_{j} B_{i}\right)
$$

The covariant form of this interaction is

$$
H=-\frac{1}{2} \mathcal{M} W_{\mu}^{*} W_{\nu}\left(\partial_{\mu} \tilde{F}_{\nu \alpha}+\partial_{\nu} \tilde{F}_{\mu \alpha}\right) k_{\alpha}
$$

Here $k$ is the 4-momentum of the W-boson, $W_{\mu}=e_{\mu} / \sqrt{2 k_{0}}, e_{\mu}=\left(e_{0}, \mathbf{e}\right)$ is its covariant polarization vector, $\partial_{\mu}=\partial / \partial x_{\mu}, \tilde{F}_{\mu \nu}=\frac{1}{2} \epsilon_{\mu \nu \alpha \beta} F_{\alpha \beta}$.

3. We are going over now to the direct calculation of the W-boson MQM in the standard model to two-loop approximation. The general structure of the diagrams which could contribute to the effect in that approximation is presented in Fig.1. The external photon line can be attached on to any quark or W propagator. To first order in the external photon momentum $q$, which corresponds to the EDM interaction with a constant external field, the sum of those diagrams vanishes [2]. Now however we are interested in the terms of second order in $q$.

The CP-odd part of the loop flavour structure is:

$$
\begin{aligned}
& i \tilde{\delta}[d(c(b-s) t-t(b-s) c+t(b-s) u-u(b-s) t+u(b-s) c-c(b-s) u) \\
& +s(c(d-b) t-t(b-s) c+t(d-b) u-u(d-b) t+u(d-b) c-c(d-b) u) \\
& +b(c(s-d) t-t(s-d) c+t(s-d) u-u(s-d) t+u(s-d) c-c(s-d) u)]
\end{aligned}
$$

For the KM matrix we use the standard parametrization of Ref.[6] where the CP-odd invariant is

$$
\tilde{\delta}=\sin \delta c_{1} c_{2} c_{3} s_{1}^{2} s_{2} s_{3} .
$$

The letters $u, d, s, c, b, t$ denote here the Green's functions of the corresponding quarks. Each product of four quark propagators allows for cyclic permutations of the kind

$$
u d c s=d c s u=c s u d=s u d c .
$$

From expression (6) it follows in particular that any diagram should be antisymmetrized in the masses $m_{1}$ and $m_{3}$ of the quarks adjoint to its upper block. This upper block can be either mass or vertex operator depending on where the photon is attached to. This antisymmetry property is sufficient for vanishing of the first term of the expansion in the photon momentum $q$, this term corresponding to EDM [2]. We will demonstrate below that the next term of the expansion in $q$ does not turn to zero after the antisymmetrization and summation over all flavors.

From all the possibilities of the photon attachment presented in Figs.2 - 8, diagrams 5 - 8 only can contribute to the effect. Diagrams 2 - 4 vanish after the antisymmetrization in the masses $m_{1}$ and $m_{3}$. Let us determine first the spinor structure of the upper part of the quark loop in diagrams $5-8$, the mass or vertex operator together with both adjoint quark 
propagators. The left-handed projectors $1+\gamma_{5}$ present in the weak interaction vertices, single out in it vector or axial structure only. The type of the MQM interaction with the electromagnetic field gradient (5) we are looking for, fixes the general structure of that part (up to multiplying by $\gamma_{5}$ ) as:

$$
h\left(p^{2}\right)(p q) \gamma_{\mu} p_{\nu} \tilde{F}_{\mu \nu}
$$

where $p$ is the momentum inside the quark loop (see Fig.5) and $h\left(p^{2}\right)$ is an invariant function dependent on $p^{2}$ and quark masses squared. It can be easily checked that after contracting with the $\gamma$-matrix structure left and integrating over $p$ expression (8) produces indeed stucture (5) we are looking for.

Let us consider now in more detail how structure (8) arises. The complete expression for the upper part of diagrams $5-8$ can be written as follows:

$$
\begin{array}{r}
A_{\mu}\left(1+\gamma_{5}\right)\left[e_{1} S_{1}(p-q) \gamma_{\mu} S_{1}(p) \Sigma(p) S_{3}(p)+S_{1}(p-q) \Gamma_{\mu}(p-q, p) S_{3}(p)\right. \\
\left.+e_{1} S_{1}(p-q) \Sigma(p-q) S_{3}(p-q) \gamma_{\mu} S_{3}(p)\right]\left(1-\gamma_{5}\right) .
\end{array}
$$

Here $A_{\mu}$ is the electromagnetic vector-potential, $e_{1}$ is the charge of the quark with mass $m_{1} ; S_{i}(p)=\left(\hat{p}-m_{i}\right)^{-1}$. The mass operator and the vertex part are denoted as $\Sigma$ and $\Gamma_{\mu}$ respectively. The projectors $1 \pm \gamma_{5}$ originate from the external W-boson interaction vertices.

The zeroth and first terms of the expansion of expression (9) in $q$ vanish after the antisymmetrization in the indices 1 and 3 [2]. The second term of the expansion consists of several contributions. It should be noted first of all that the contribution corresponding to the second-order term in the expansion of $\Gamma_{\mu}$ in $q$ vanishes. Indeed, it is symmetric in the masses $m_{1}$ and $m_{3}$ since in the propagator $S_{1}$ the momentum $q$ can be put equal to zero. The contributions left can be conveniently split into two groups. To the first one belong those terms where the first term of the $\Gamma_{\mu}$ expansion in $q$ is multiplied by the first term in the expansion of the propagator $S_{1}$. To the second one belong all the contributions with the mass operator and vertex part taken at the vanishing $q$.

The first group of contributions does not need renormalization and the corresponding interim result can be obtained rather easily:

$$
\begin{array}{r}
A_{\mu}\left(1+\gamma_{5}\right)\left[\left.\frac{\hat{p}-\hat{q}}{(p-q)^{2}-m_{1}^{2}} q_{\nu} \frac{\partial \Gamma_{\mu}}{\partial q_{\nu}}\right|_{q=0} \frac{\hat{p}}{p^{2}-m_{3}^{2}}\right]\left(1-\gamma_{5}\right)-\left(m_{1} \leftrightarrow m_{3}\right) \\
\longrightarrow \frac{2 e_{2}\left(m_{1}^{2}-m_{3}^{2}\right) h\left(p^{2}\right) p^{2}(p q) \gamma_{\mu} p_{\nu} \tilde{F}_{\mu \nu}}{\left(p^{2}-m_{1}^{2}\right)^{2}\left(p^{2}-m_{3}^{2}\right)^{2}}\left(1-\gamma_{5}\right) .
\end{array}
$$

Here $h\left(p^{2}\right)$ denotes a result of the loop integration which will be explicitly calculated below, $e_{2}$ is the charge of the quark inside the vertex.

To investigate the second group of contributions we will need expressions for the mass operator and vertex part [1, 2]. Unrenormalized mass operator in the V-A theory is simple:

$$
\Sigma=\hat{p}\left(1+\gamma_{5}\right) f\left(p^{2}\right)
$$

The renormalization introduces into the operator $\Sigma$ the dependence on external masses:

$$
\Sigma=\hat{p}\left(1+\gamma_{5}\right) \tilde{f}\left(p^{2}\right)-f_{13}\left[\hat{p}\left(1-\gamma_{5}\right)-m_{1}\left(1-\gamma_{5}\right)-m_{3}\left(1+\gamma_{5}\right)\right],
$$


where $f_{13}$ and $\tilde{f}$ are expressed via the function $f$ and masses $m_{1}, m_{3}$ as follows:

$$
\tilde{f}\left(p^{2}\right)=f\left(p^{2}\right)-\frac{m_{1}^{2} f_{1}-m_{3}^{2} f_{3}}{m_{1}^{2}-m_{3}^{2}}, \quad f_{13}=\frac{m_{1} m_{3}\left(f_{1}-f_{3}\right)}{m_{1}^{2}-m_{3}^{2}} ; \quad f_{i}=f\left(p^{2}=m_{i}^{2}\right), \quad i=1,3 .
$$

The vertex part renormalization at the vanishing momentum of external photon should comply with the Ward identity:

$$
\Gamma_{\mu}(p, p)=-e_{1} \frac{\partial \Sigma}{\partial p_{\mu}}
$$

where $e_{1}$ is the charge of the quark with mass $m_{1}$. This identity allows one to reduce the second group of contributions to

$e_{1} A_{\mu}\left(1+\gamma_{5}\right)\left[S_{1}(p-q) \gamma_{\mu} S_{1}(p) \Sigma(p) S_{3}(p)-S_{1}(p-q) \frac{\partial \Sigma}{\partial p_{\mu}} S_{3}(p)+S_{1}(p-q) \Sigma(p-q) S_{3}(p-q) \gamma_{\mu} S_{3}(p)\right]\left(1-\gamma_{5}\right)$.

The substitution of the renormalized operator $\Sigma$ into this expression and its expansion in $q$ leads to a simple result. The sum of the terms proportional to $f_{13}$ is symmetric in the masses and therefore does not contribute to the effect. A nonvanishing contribution originates from that term in the mass operator which is proportional to $\hat{p}\left(1+\gamma_{5}\right)$. Omitting intermediate steps, we obtain for the second group contribution the following expression:

$$
\frac{2\left(m_{1}^{2}-m_{3}^{2}\right) \tilde{f}^{\prime} p^{2}(p q) \gamma_{\mu} p_{\nu} \tilde{F}_{\mu \nu}}{\left(p^{2}-m_{1}^{2}\right)^{2}\left(p^{2}-m_{3}^{2}\right)^{2}}\left(1-\gamma_{5}\right)
$$

where $\tilde{f}^{\prime}=\partial \tilde{f} / \partial\left(p^{2}\right)$. Curiously enough, the renormalization counterterms do not contribute at all to the effect the derivative of $\tilde{f}$ in $p^{2}$ coincides with that of the unrenormalized function $f$ :

$$
\frac{\partial \tilde{f}}{\partial\left(p^{2}\right)}=\frac{\partial f}{\partial\left(p^{2}\right)}
$$

Both expressions, (10) and (15), produce contributions to the W-boson MQM. Indeed, their contraction with the gamma-matrix structure left allows one to write down the effective interaction of the electromagnetic field gradient with W-boson as follows:

$$
H_{\text {eff }}=\tilde{\delta} \frac{g^{4}}{2} W_{\mu}^{*} W_{\nu} \sum_{\text {flavour }} \int \frac{d^{4} p}{(2 \pi)^{4}} \frac{(p q) p_{\beta}\left(p_{\nu} \tilde{F}_{\mu \beta}+p_{\mu} \tilde{F}_{\nu \beta}\right)\left(m_{1}^{2}-m_{3}^{2}\right)\left[e_{1} f^{\prime}+e_{2} h\right] p^{2}}{\left(p^{2}-m_{1}^{2}\right)^{2}\left(p^{2}-m_{3}^{2}\right)^{2}\left[(k-p)^{2}-m_{4}^{2}\right]} .
$$

In this expression $g$ is the semiweak charge, $m_{4}$ is the mass of the quark in the lower fermion line. One can easily check that after the integration over $p$ interaction (5) arises. Indeed, in the result $(p q) p_{\beta} p_{\nu(\mu)}$ is substituted for by $q_{\nu(\mu)} k_{\beta}$ which reduces (16) to (5). The exact cancellation of contributions (10) and (15) is impossible since the functions $h\left(p^{2}\right)$ and $f^{\prime}\left(p^{2}\right)$ depend differently on $p^{2}$ and the masses of the quarks inside the mass operator and vertex part.

4. After convincing ourselves in the absence of the exact cancellation of the W-boson MQM in two-loop approximation, we are going to find its value. 
It is natural to consider all quark masses but $m_{t}$ small as compared to the W-boson one $M$. Together with the quark mass hierarchy it allows one to simplify the calculations considerably, restricting to those contributions to MQM which are of lowest order in the light quark masses. Besides, it is also natural to single out the contributions with logarithms of large mass ratios, e.g., $\log \left(m_{t} / m_{c}\right), \log \left(m_{b} / m_{s}\right), \log \left(M / m_{b}\right)$ etc.

All the diagrams can be split into two types, depending on which quarks, $U(u, c, t)$ or $D(d, s, b)$, flow inside the mass or vertex operator. It is convenient to sum first of all over the flavours of the quarks masses of which were denoted up to now as $m_{1}$ and $m_{3}$. For the two types mentioned we get respectively:

$$
\begin{aligned}
\sum \frac{\left(m_{1}^{2}-m_{3}^{2}\right)}{\left(p^{2}-m_{1}^{2}\right)^{2}\left(p^{2}-m_{3}^{2}\right)^{2}} \longrightarrow & \frac{-m_{b}^{4} m_{s}^{2}}{p^{4}\left(p^{2}-m_{b}^{2}\right)^{2}\left(p^{2}-m_{s}^{2}\right)^{2}} \\
& \frac{-m_{t}^{4} m_{c}^{2}}{p^{4}\left(p^{2}-m_{t}^{2}\right)^{2}\left(p^{2}-m_{c}^{2}\right)^{2}} .
\end{aligned}
$$

In expression (17) we put $m_{u}=m_{d}=0$. We can determine now the characteristic momenta $p$. When quarks are arranged according to the second line of formula (17), integral (16) is infrared divergent if one neglects the masses $m_{s}$ and $m_{b}$ in the denominator. It means that the typical loop momenta contributing to the effect are $p \sim m_{b}$. In the opposite case when D-quarks are inside the mass or vertex operator, the typical momenta range is large: $p \sim M$.

Now we have to sum over the flavors left. Here the difference is essential in the dependence of $f^{\prime}\left(p^{2}\right)$ and $h\left(p^{2}\right)$ on quark masses. The function $f$ allows for the expansion in the mass for the case of a light quark inside the mass operator:

$$
f^{\prime}\left(p^{2}, m^{2}\right)=f^{\prime}\left(p^{2}, m^{2}=0\right)+\left.m^{2} \frac{d f^{\prime}}{d\left(m^{2}\right)}\right|_{m=0}+\ldots
$$

We neglect the terms of higher order in a light quark mass. For D-quarks inside the mass operator one can easily demonstrate the cancellation of the terms of zeroth, second and fourth powers in mass:

$$
\left.\frac{f^{\prime}\left(m_{b}^{2}\right)-f^{\prime}\left(m_{s}^{2}\right)}{(k-p)^{2}-m_{d}^{2}}+\frac{f^{\prime}\left(m_{s}^{2}\right)-f^{\prime}\left(m_{d}^{2}\right)}{(k-p)^{2}-m_{b}^{2}}+\frac{f^{\prime}\left(m_{d}^{2}\right)-f^{\prime}\left(m_{b}^{2}\right)}{(k-p)^{2}-m_{s}^{2}}=\mathcal{O}\left(m_{b}^{4} m_{s}^{2}\right)\right)
$$

Therefore this contribution to the MQM is $\mathcal{O}\left(m_{b}^{4} m_{c}^{2} m_{s}^{2}\right)$, i.e., it is suppressed as the eighth power of the ratio of light quark masses to that of $\mathrm{W}$. In the opposite group of diagrams one cannot expand in $m_{t}$, so the summation left introduces the suppression $\sim m_{c}^{2}$ :

$$
\sum \frac{f^{\prime}\left(p^{2}, m^{2}\right)}{\left.(k-p)^{2}-m_{4}^{2}\right)} \longrightarrow-\left.\frac{d f^{\prime}}{d\left(m^{2}\right)}\right|_{m=0} \frac{m_{c}^{2}}{(k-p)^{2}-m_{t}^{2}}+f^{\prime}\left(m_{t}^{2}\right) \frac{m_{c}^{2}}{(k-p)^{4}}
$$

Since the integral over $p$ is dominated by momenta $p \sim m_{b}$ (see formula (17)), the discussed contribution to the MQM is $\mathcal{O}\left(m_{b}^{2} m_{c}^{2} m_{s}^{2}\right)$. However, we will not to calculate it since this contribution is small as compared with another term in expression (16) which contains the function $h\left(p^{2}, m^{2}\right)$.

The point is that, as distinct from the function $f, h$ does not allow for a simple expansion in a light quark mass. For a light quark inside the vertex part, $h$ contains terms proportional 
to $m^{2} \log m^{2}$. Therefore, the terms containing $m_{s}^{2} m_{b}^{2}$ in the expression analogous to (19), not only do not cancel, but are enhanced as $\log \left(m_{b}^{2} / m_{s}^{2}\right)$. Let us demonstrate this assertion in more detail. To this end we write down the vertex integral explicitly (in fact its dominating part where the quark interacts with the external field) and sum over the masses of all quarks in the upper and lower propagators of the quark loop. The result is:

$$
\frac{g^{2} e_{2} m_{s}^{2} m_{b}^{6}}{\left((k-p)^{2}-m_{s}^{2}\right)\left((k-p)^{2}-m_{b}^{2}\right)} \int \frac{d^{4} l}{(2 \pi)^{4}} \frac{\tilde{F}_{\mu \nu}\left(\gamma_{\mu} l_{\nu}+\frac{2}{M^{2}} \hat{l}_{\mu} p_{\nu}\right)\left(1+\gamma_{5}\right)}{l^{2}\left(l^{2}-m_{s}^{2}\right)^{2}\left(l^{2}-m_{b}^{2}\right)^{2}\left[(p-l)^{2}-M^{2}\right]}
$$

It is obvious now that the integral is dominated by the momenta region $m_{s} \ll l \ll m_{b}$ and contains a large logarithm. Expanding the denominator in the ratio $(p l) /\left(p^{2}-M^{2}\right)$ we obtain

$$
\frac{g^{2} e_{2} m_{s}^{2} m_{b}^{2} \log \left(m_{b}^{2} / m_{s}^{2}\right)}{16 \pi^{2}\left((k-p)^{2}-m_{s}^{2}\right)\left((k-p)^{2}-m_{b}^{2}\right)}\left[\frac{1}{\left(p^{2}-M^{2}\right)^{2}}+\frac{1}{p^{2}-M^{2}}\right] \tilde{F}_{\mu \nu} \gamma_{\mu} p_{\nu}\left(1+\gamma_{5}\right) .
$$

This formula is valid for all $p^{2}$ as long as $p^{2}-M^{2} \gg M m_{b}$.

The last integral over $p$ contains also a large logarithm when W-boson is on mass shell. It can be easily checked indeed that the integral diverges logarithmically if one neglects quark masses in the denominator of the factor preceding the integral in formula (21) and put $k^{2}=M^{2}$. Retaining in the last integral that contribution only which is enhanced by a large logarithmic factor $\log \left(M^{2} / m_{b}^{2}\right)$, we obtain the final expression for the CP-odd interaction of $\mathrm{W}$-boson with the electromagnetic field gradient:

$H_{e f f}=\tilde{\delta} \frac{e g^{4}}{12\left(16 \pi^{2}\right)^{2}} \frac{m_{s}^{2} m_{c}^{2} m_{b}^{2}}{M^{4}} \frac{m_{t}^{4}}{\left(m_{t}^{2}-M^{2}\right)^{2}} \log \left(m_{b}^{2} / m_{s}^{2}\right) \log \left(M^{2} / m_{b}^{2}\right) W_{\mu}^{*} W_{\nu}\left(\partial_{\mu} \tilde{F}_{\nu \alpha}+\partial_{\nu} \tilde{F}_{\mu \alpha}\right) k_{\alpha}$.

As to another group of diagrams, with $U$-quarks in the vertex part, they also contain $\log \left[\left(M^{2}-p^{2}\right) / m_{c}^{2}\right]$ in a formula analogous to (22), but cannot compete with (23) since they have no $\log$ in the integral over $p^{2}$.

Going over to the Fermi constant $G_{F}=\sqrt{2} g^{2} /\left(8 M^{2}\right)$ we get the final formula for the W-boson MQM in the Kobayashi-Maskawa model:

$$
\mathcal{M}=-\frac{e}{48 \pi^{4}} \tilde{\delta} G_{F}^{2} \frac{m_{s}^{2} m_{c}^{2} m_{b}^{2}}{M^{4}} \frac{m_{t}^{4}}{\left(m_{t}^{2}-M^{2}\right)^{2}} \log \left(m_{b}^{2} / m_{s}^{2}\right) \log \left(M^{2} / m_{b}^{2}\right) .
$$

The extreme smallness of the MQM in the standard model is in no way unexpected. In other models of CP-violation $\mathcal{M}$ can well turn out much larger.

We are greatly indebted to C.P. Burgess, C. Hamzaoui, G. Couture, G. Jakimow and I. Maksymyk for stimulating discussion and correspondence. 


\section{References}

[1] E.P. Shabalin. Yad.Fiz. 28 (1978) 151 (Sov.J.Nucl.Phys. 28 (1978) 75)

[2] M.E. Pospelov and I.B. Khriplovich. Yad.Fiz. 53 (1991) 1030 (Sov.J.Nucl.Phys. 53 (1991) 638)

[3] D. Chang, W.Y. Keung and J. Liu. Nucl.Phys. B 355 (1991) 295

[4] I.Yu. Kobzarev, L.B. Okun' and M.V. Terentyev. Pis'ma v ZhETF 2 (1965) 466 (Sov.Phys.JETP Lett. 2 (1965) 289)

[5] V.B. Beresetetsky, E.M. Lifshitz and L.P. Pitaevsky. Quantum Electrodynamics (Pergamon Press, Oxford 1982)

[6] L.B. Okun'Leptons and Quarks (Nauka, Moscow 1981) 


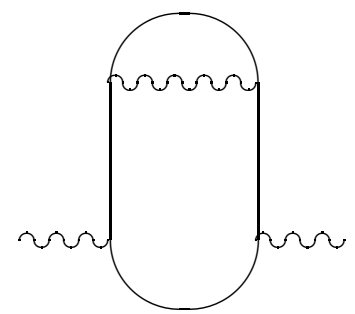

Fig. 1

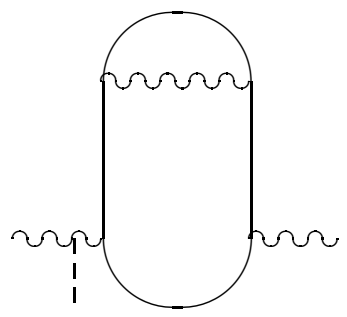

Fig. 2

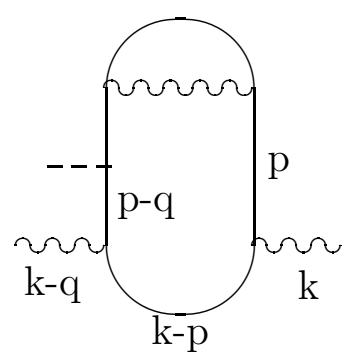

Fig. 5

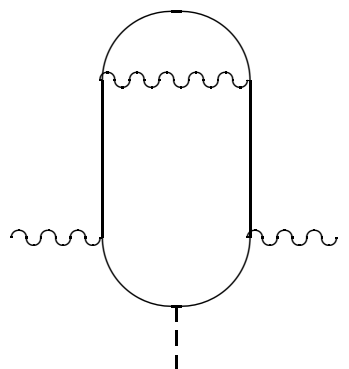

Fig. 3

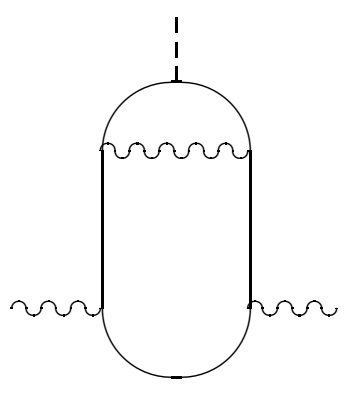

Fig. 6

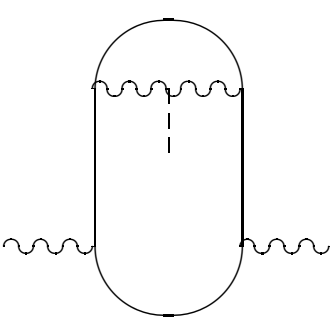

Fig. 7

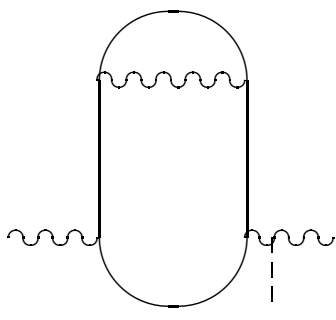

Fig. 4

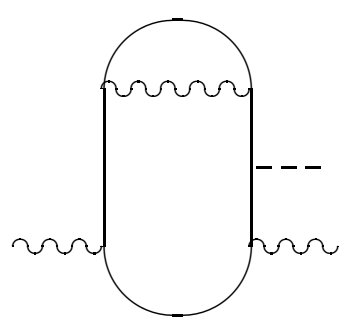

Fig. 8 